\title{
Évolutions récentes de la conditionnalité des prestations sociales en Australie
}

Tamara Walsh

\section{(2) OpenEdition}

1 Journals

\section{Édition électronique}

URL : https://journals.openedition.org/rdctss/1684

DOI : $10.4000 /$ rdctss. 1684

ISSN : 2262-9815

Éditeur

Centre de droit comparé du travail et de la sécurité sociale

\section{Édition imprimée}

Date de publication : 1 avril 2019

Pagination : 194-197

ISSN : 2117-4350

\section{Référence électronique}

Tamara Walsh, «Évolutions récentes de la conditionnalité des prestations sociales en Australie»,

Revue de droit comparé du travail et de la sécurité sociale [En ligne], 1 | 2019, mis en ligne le 01 novembre 2021, consulté le 13 novembre 2021. URL : http://journals.openedition.org/rdctss/1684 ; DOI : https:// doi.org/10.4000/rdctss. 1684

\section{(c) $(7)(9$}

Revue de droit comparé du travail et de la sécurité sociale est mise à disposition selon les termes de la Licence Creative Commons Attribution - Pas d'Utilisation Commerciale - Pas de Modification 4.0 International. 


\section{EVOLUTIONS RÉCENTES DE LA CONDITIONNALITÉ DES PRESTATIONS SOCIALES EN AUSTRALIE}

L'octroi des prestations de sécurité sociale et d'autres formes de protection sociale a toujours été assujetti à des conditions d'attribution. Si certains soutiennent que la protection sociale devrait être " universelle " ${ }^{1}$, la plupart convienne que l'imposition de certaines conditions limitant l'éligibilité ou le montant des prestations reçues est appropriée ${ }^{2}$. Cependant, des commentateurs soulèvent actuellement des objections, en Australie, car les conditions imposées aux bénéficiaires de la sécurité sociale ont de plus en plus un caractère " comportemental» et racialisé. En plus de satisfaire à certaines conditions de résidence, de moyens et de biens matériels, les bénéficiaires de la sécurité sociale sont désormais tenus de prendre des décisions différentes en termes de mode de vie, sous peine de sanction pour non-conformité.

\section{I - « L'ANCIENNE » CONDITIONNALITÉ DANS LA LÉGISLATION AUSTRALIENNE SUR LA SÉCURITÉ SOCIALE}

Dans la législation australienne sur la sécurité sociale, une distinction est établie entre les « allocations " et les "pensions " ${ }^{3}$, à la fois en termes de conditions de qualification et de montant des paiements. Les «pensions » sont celle de vieillesse et celle d'aide aux personnes handicapées. Elles sont versées à des taux plus élevés que les "allocations». Celles-ci comprennent les allocations de chômage (allocation Newstart pour adultes et allocation jeune pour les jeunes), les allocations pour responsabilités parentales et les allocations versées aux aidants.

Une condition de résidence s'applique à toutes les prestations de sécurité sociale, et la plupart des allocations de soutien du revenu ont une période d'attente de deux ou quatre ans pour les résidents nouvellement arrivé 4 . Des exceptions peuvent s'appliquer dans certaines circonstances, par exemple pour les parents célibataires et les réfugiés ${ }^{5}$. Outre les conditions de résidence, toutes les allocations de soutien du revenu sont soumises à une condition de ressources. Ces critères ont été qualifiés de "strictement arithmétiques " ${ }^{6}$, en ce sens que ceux dont le revenu ou les actifs dépassant une limite de valeur définie ne sont pas admis à l'allocation et un taux forfaitaire est versé. Le critère des moyens et des biens matériels s'applique également aux pensions, mais les montants des prestations sont pondérés et calculés à l'aide d'une formule spécifique tenant compte de leurs revenus et de leurs actifs. En outre, certains actifs sont exonérés?

D. Beland, P. Blomqvist, J. Goul Andersen, J. Palme, A. Waddan, "The universal decline of universality? Social policy change in Canada, Denmark, Sweden and the UK", Social Policy and Administration, 2014, vol.48, n 7 , p. 739.

2 T. Eardley, P. Saunders, C. Evans, Community attitudes towards unemployment, activity testing and mutual obligation, Social Policy Research Centre Document de discussion, n 107, 2000; C. Mclntyre, "From entitlement to obligation in the Australian welfare state", Australian Journal of Social Issue, 1999, vol. 34, n² 2, p. 103.

3 Communément appelées « prestations de sécurité sociale », Voir Social Security Act 1991 (Cth) s23.

4 Le $1^{\text {er }}$ janvier 2019, la période de carence de la plupart des prestations de soutien du revenu a été augmentée et passe de deux à quatre ans, Social Security Act 1991 (Cth) ss 7(4A), 23(1), 623A-623B.

5 Social Security Act 1991 (Cth) ss 7(6), 500X (3).

6 T. Carney, "Social security law: What does the politics of "conditional welfare" mean for review and client representation?", Australian Journal of Social Issues, 2011, vol. 46, n 3, p. 233.

7 II s'agit du "Pension Rate Calculator", Social Security Act 1991 (Cth) s 55. 
Une personne qui demande une pension d'invalidité doit faire évaluer l'invalidité qui la rend inapte à travailler pendant 15 heures par semaine au maximum ${ }^{8}$. Pour être admissible, une personne doit « marquer » au moins 20 points dans les «tableaux des handicaps ". Ces tableaux étant régis par une décision ministérielle, ils peuvent être modifiés à tout moment par le ministre compétent, sans le contrôle du Parlement ${ }^{10}$. La dernière fois qu'ils ont été modifiés, en 2011, on a estimé qu'environ quatre bénéficiaires sur dix allaient immédiatement devenir inéligibles ${ }^{11}$. Au cours des dernières années, il y a eu une réduction importante du nombre de personnes jugées admissibles à la pension d'aide aux personnes handicapées ${ }^{12}$. En effet, l'an dernier plus de 60 $\%$ des personnes ayant demandé une pension d'aide aux personnes handicapées ont vu leur demande rejetée ${ }^{13}$. Ceux jugés inéligibles pour la pension d'aide aux personnes handicapées sont obligés de compter sur l'allocation Newstart, une indemnité de chômage, ce qui signifie qu'ils reçoivent un montant bien moins élevé ${ }^{14}$, et qu'ils sont soumis à des "conditions de participation » strictes.

\section{II - LES CONDITIONS LIÉES AU TRAVAIL: DE « L'ANCIENNE » À LA « NOUVELLE » CONDITIONNALITÉ}

Depuis longtemps, des conditions très strictes et liées au travail sont imposées aux bénéficiaires d'allocations de chômage en Australie ${ }^{15}$. Mais récemment, le nombre et la complexité de ces conditions ont considérablement augmenté. Le non-respect des « conditions de participation", telles que la participation à des entretiens d'embauche, le contact avec Centrelink et l'acceptation de toutes les offres d'emploi « appropriées », peut désormais entraîner des suspensions d'allocation de quatre à six semaines, des réductions ou des suppressions de l'allocation en cas de non-respect persistant ${ }^{16}$. Si une personne démontre qu'elle avait une excuse raisonnable pour ne pas s'y conformer, elle peut obtenir un paiement rétroactif. Cependant la suspension de son allocation pendant plusieurs semaines peut avoir à court terme des conséquences graves difficilement réversibles, comme une détérioration de sa santé physique et mentale ou une rupture du bail de location ${ }^{17}$.

Jusqu'à récemment, les personnes handicapées et celles qui avaient des responsabilités familiales étaient dispensées de l'obligation de participation, mais ce n'est plus le cas. En 2014, les bénéficiaires d'une pension d'aide aux personnes handicapées ont dû pour la première fois

8 Social Security Act 1991 (Cth) s 94(1).

9 Social Security Act 1991 (Cth) s 26.

10 Social Security (Tables for the Assessment of Work-related Impairment for Disability Support Pension) Determination 2011.

11 S. Lunn, "Gillard tightens disability pensions" The Australian, 30 July 2011.

12 National Welfare Rights Network, 'Hundred lose pension as Impairment Tables changed', Welfare Rights Review, 2017, vol. 1, n³, p. 1; Parliamentary Budget Office, Disability Support Pension: Historical and Projected Trends, Parlement d'Australie, Rapport n 1, 2018.

13 J. Norman, "New Disability Support Pension recipients down by almost 60,000, due to Gillard-led crackdown", ABC News, 21 février 2018.

14274 \$AUD par semaine, contre 448 \$AUD pour la pension de vieillesse: ACOSS, 'Disability Support Pension cuts bad news for people affected', communiqué de presse, 21 février 2018.

15 T. Walsh, G. Marston, "Benefit overpayment, welfare fraud and financial hardship", Journal of Social Security Law, 2010, vol.1, n², p. 100; T. Carney, "Welfare to work: Or work discipline revisited', Australian Journal of Social Issues, 2006, vol. 41, n 1, p. 27.

16 Social Security (Administration) Act 1999 (Cth) ss 42AA-42AP.

17 T. Eardley, J. Brown, M. Rawsthorne, K. Norris, L. Emrys, The Impact of Breaching on Income Support Customers, Social Policy Research Centre, 2005; S. Schooneveldt, "Do mutual obligation breach penalties coerce compliance with government expectations?", Australian Journal of Social Issues, 2004, vol.39, n², p. 155. 
souscrire à un " plan de participation » les obligeant à s'engager dans des activités obligatoires liées à l'emploi telles que la recherche d'un emploi, les stages, l'éducation et de formation ${ }^{18}$. En 2013, des conditions de participation des « prestations sociales au travail » ont été imposées à tous les parents célibataires d'enfants âgés de huit ans au minimum et aux parents d'enfants âgés de six ans au minimum ${ }^{19}$. Et en 2016, le programme Parents Next a été introduit, imposant des conditions de participation obligatoires, y compris la participation à des « séances de lecture " à la bibliothèque, à des cours de natation et à des groupes de jeu, aux parents dont le plus jeune enfant a entre six mois et cinq ans ${ }^{20}$.

\section{III - LA « NOUVELLE » CONDITIONNALITÉ: LES CONDITIONS LIÉES AU COMPORTEMENT}

Outre le développement sans précédent des conditions liées au travail, de nombreuses obligations ont également été introduites, pouvant être qualifiées de "comportementales", c'est-à-dire que leur objectif est d'obliger les bénéficiaires de la sécurité sociale à se conformer à certaines normes sociales, ou à remplir certaines " conditions de vie contraignantes ${ }^{21}$, sous peine de non-paiement des prestations. Ces réformes ont en particulier mis l'accent sur le comportement des parents, ce qui se justifie par des préoccupations relatives au bien-être des enfants dans certaines zones défavorisées, en particulier les communautés autochtones ${ }^{22}$.

La série de réformes la plus importante incorporant cette "nouvelle » conditionnalité dans la législation australienne sur la sécurité sociale était celle associée à l'intervention nationale d'urgence de 2007 sur le territoire du Nord ${ }^{23}$. Une des mesures portait sur la " gestion des revenus ", en vertu de laquelle une partie de l'allocation de soutien du revenu d'une personne (habituellement $50 \%$ ou $70 \%$ ) est placée sous séquestre et réservée exclusivement à l'achat de biens considérés comme des «besoins prioritaires». Cette partie de l'allocation est versée sur une carte de débit, appelée "BasicsCard», qui ne peut être utilisée que dans certains magasins pour certains biens et services, et qu'il est impossible d'utiliser pour acheter du tabac, de l'alcool, des jeux de hasard ou de la pornographie ${ }^{24}$. La gestion du revenu a d'abord été limitée à un nombre distinct de communautés autochtones aborigènes du Territoire du Nord, considérées comme particulièrement dysfonctionnelles, mais nombre de ces mesures ont depuis été étendues ou déployées plus largement. En vertu de la législation actuellement en vigueur, une personne peut être soumise à la gestion obligatoire de ses revenus si elle est considérée comme un " jeune désengagé » ou un " bénéficiaire de l'aide sociale à long terme ", si elle est " vulnérable " en raison d'un problème de santé mentale ou du fait qu'elle est sans abri, si elle a été signalée aux autorités de protection de l'enfance ou si elle a des enfants qui ne sont pas inscrits ou qui ne vont pas régulièrement à l'école ${ }^{25}$. Le nombre de sites "d'essai » sur lesquels ces mesures

18 Social Security Act 1991 (Cth) s 94A, 94B. L. Buckmaster, "Changes to Disability Support Pension: Budget Review 2014-15", 2014, Parlement d'Australie.

19 Social Security Act 1991 (Cth) s 500A, 500D.

20 Social Security (Parenting payment participation requirements - classes of persons) Specification 2016 $\left(n^{\circ} 1\right)$. Ce programme a été élargi en 2018: L. Henriques-Gomes, "Single parents forced to attend "story time" or lose Centrelink payments", The Guardian, 6 novembre 2018.

21 T. Carney, "Social security law: What does the politics of "conditional welfare" mean for review and client representation?", (2011) 46(3) Australian Journal of Social Issues, 2011 ; vol.46, n 3, p. 233.

22 Voir en particulier D. R Taylor, M. Gray, D. Stanton, "New conditionality in Australian social security policy" Australian Journal of Social, 2016, vol. 51, n 1, Issues 3.

23 Northern Territory National Emergency Response Act 2007 (Cth).

24 Social Security (Adminisration) Act 1999 (Cth) ss 123TA-123UGA.

25 Social Security (Administration) Act 1999 (Cth) ss 123UA-123UGA. 
s'appliquent a régulièrement augmenté depuis 2010 et tout indique que le nombre de sites sur lesquels elles s'appliqueront continuera à augmenter.

\section{IV - LES IMPACTS DE LA « NOUVELLE » CONDITIONNALITÉ}

La tendance à la conditionnalité de l'aide sociale a été critiquée par le secteur des services sociaux car elle était fondée sur des notions dépassées de " mérite » et de " déviance » et qu'elle était vécue comme dégradante et humiliante par ceux qui la subissaient ${ }^{26}$. Ils estiment que l'aide sociale conditionnelle enfreint les droits fondamentaux des individus à la liberté, à l'autonomie et à l'autodétermination ${ }^{27}$. Ils soulignent que l'aide sociale conditionnelle a eu peu de succès puisque le taux d'emploi des personnes handicapées n'a pas augmenté et qu'aucune amélioration mesurable de la sécurité des enfants n'a été apportée sans la mise en place de services d'aide familiale intensive ${ }^{28}$.

D'autres conséquences négatives de la conditionnalité peuvent être plus difficiles à gérer. Par exemple, les personnes peuvent être réticentes à demander de l'aide si elles savent que les travailleurs avec lesquels elles interagissent auront la responsabilité de faire un rapport sur leurs "progrès» et leur niveau de conformité29. En outre, les périodes de non-paiement des prestations peuvent avoir de graves conséquences et entraîner une criminalisation lorsqu'une personne se sent obligée de se livrer à des activités illégales pour survivre ${ }^{30}$. Les effets peuvent donc être néfastes non seulement pour la personne elle-même, mais pour toutes les personnes à sa charge.

Les bénéficiaires de la sécurité sociale ne sont généralement pas hostiles aux modifications du système de sécurité sociale, si celles-ci ont pour effet de les aider à trouver un emploi ou de leur fournir un soutien supplémentaire en matière de services sociaux. Ce qu'ils espèrent, c'est un système suffisamment souple pour évaluer leurs besoins individuels et fournir un soutien individualisé permettant d'y répondre. Les personnes interrogées affirment systématiquement qu'elles veulent simplement être traitées avec dignité et respect, qu'on les voit comme des personnes plutôt que comme des problèmes et qu'on leur manifeste de l'empathie ${ }^{31}$. C'est bien le moins que les bénéficiaires de la sécurité sociale puissent attendre de leur gouvernement.

26 K. Soldatic, B. Pini "The three Ds of welfare reform: Disability, disgust and deservingness", Australian Journal of Human Rights, 2009, vol. 15, n¹, p. 77.

27 A. Nevile, "Human rights, power and welfare conditionality", Australian Journal of Human Rights, 2009, vol.14, $n^{\circ} 1$, p. 1; S. Bielefeld, "Compulsory income management, Indigenous peoples and structural violence: Implications for citizenship and autonomy", Australian Indigenous Law Review, 2014, vol. 18, $\mathrm{n}^{\circ} 1$, p. 99.

28 J. R. Bray, M. Gray, K. Hand, B. Bradbury, Ch. Eastman I. Katz, Evaluating New Income Management in the Northern Territory: First Evaluation Report, Social Policy Research Centre and ANU, 2012; K. Hand, I. Katz, J R. Bray, "Welfare conditionality as a child protection tool", Family Matters, 2016, n 97, p. 16; J. Ireland, "Evidence does back claims of a blow-out in numbers receiving disability support pension", Sydney Morning Herald, 23 mai 2014.

29 D. R. Taylor, M. Gray et D. Stanton, "New conditionality in Australian social security policy", Australian Journal of Social Issues, 2016, vol. 51, n 1, 3 p 19.; I. Shutes, R. Taylor, "Conditionality and the financing of employment services - Implications for the social divisions of work and welfare", Social Policy and Administration, 2014, vol. 48, n²; T. Carney, "Social security law: What does the politics of "conditional welfare" mean for review and client representation?", Australian Journal of Social Issues, 2011, vol. 46, $n^{\circ} 3$, p. 233.

30 Del Roy Fletcher, Sh. Wright, "A hand up or a slap down? Criminalising benefit claimants in Britain via strategies of surveillance, sanctions and deterrence", Critical Social Policy, 2018, vol. 32, n 3, p. 323.

31 A. Nevile, "Human rights, power and welfare conditionality", Australian Journal of Human Rights, 2008, vol. $14, n^{\circ} 1$ p. 7-8. 